TITLE:

\title{
Efficacy of three-dimensional endoscopy in endonasal surgery.
}

$\operatorname{AUTHOR}(S)$ :

Ogino-Nishimura, Eriko; Nakagawa, Takayuki; Sakamoto, Tatsunori; Ito, Juichi

CITATION:

Ogino-Nishimura, Eriko ...[et al]. Efficacy of three-dimensional endoscopy in endonasal surgery.. Auris Nasus Larynx 2014, 42(3): 203207

ISSUE DATE:

2014-11-04

URL:

http://hdl.handle.net/2433/198578

\section{RIGHT:}

(C) 2014 Elsevier Ireland Ltd.; 許諾条件により本文ファイルは2015-1104に公開.; この論文は出版社版でありません。引用の際には出版社版 をご確認ご利用ください。; This is not the published version. Please cite only the published version. 
Efficacy of three-dimensional endoscopy in endonasal surgery

Eriko Ogino-Nishimura, M.D. ${ }^{1,2}$; Takayuki Nakagawa, M.D., Ph.D. ${ }^{1}$; Tatsunori Sakamoto, M.D., Ph.D. ${ }^{1}$; Juichi Ito, M.D., Ph.D. ${ }^{1}$

1 Department of Otolaryngology, Head and Neck Surgery, Graduate School of Medicine, Kyoto University, Kyoto, Japan

2 Kyoto Ear, Nose, \& Throat Surgicenter, Kyotanabe, Japan

Running title: 3D endoscopy in endonasal surgery

Source of financial support: Research grant from The Japanese Foundation for Research and Promotion of Endoscopy

Corresponding Author:

Dr. Eriko Ogino-Nishimura

Kyoto Ear, Nose \& Throat Surgicenter

Yamatenishi 2-2-3, Kyotanabe,

Kyoto 610-0355, Japan

E-mail: ogino@hiroshiba.com

Tel: +81-774-64-0789, Fax: +81-774-64-0872

Foot note: This work was presented at the $2^{\text {nd }}$ Meeting of the European Academy of ORLHNS in Nice, France, April 27- 30, 2013. 


\section{ABSTRACT}

\section{Objective}

To examine the efficacy of a three-dimensional (3D) endoscope for endoscopic sinus and skull base surgery.

\section{Methods}

The study design was a retrospective case series and qualitative research. The clinical efficacy of 3D endoscopes was examined on five cadavers. We performed conventional endoscopic sinus surgery (ESS) in five cases and hypophysectomy in two cases using a 3D endoscope. The educational advantages of the 3D endoscope were assessed using questionnaires given to the participants of cadaver dissection courses.

\section{Results}

In the posterior portion of the nasal cavity, images captured via 3D endoscopy provided a superior perception of depth of information than those via two-dimensional (2D) endoscopy. All endonasal surgeries were completed in clinical settings using a 3D endoscope without perioperative complications. In terms of the operative time and amount of bleeding, the results of 3D endoscopic surgeries were not inferior to those of 2D endoscopic surgeries. Fatigue from 3D viewing through polarized glasses did not adversely affect performance of the surgery. Moreover, questionnaires for the evaluation 
of educational efficacy were completed by 73 surgeons. Of the respondents, $89 \%$ agreed that $3 \mathrm{D}$ endoscopy provided a better understanding of the surgical anatomy than did 2D endoscopy. As for the site where 3D endoscopy would be the most useful for understanding surgical anatomy, $40 \%$ of the respondents named the skull base; $29 \%$, the posterior ethmoid sinuses; and 26\%, the sphenoid sinus; and 9\%, the ethmoid bulla and middle turbinate.

\section{Conclusion}

The 3D endoscope contributes to a more precise anatomical understanding of the posterior structures of the sinuses and skull base and ensures a more precise operation of the instruments. Thus, 3D endoscopes will likely become a standard device for endonasal surgery in the near future.

\section{Key Words:}

Three-dimensional endoscopy, endoscopic sinus surgery, skull base surgery, education

Level of Evidence: N/A 


\section{INTRODUCTION}

Endoscopic surgery is a standard procedure for the treatment of sinus diseases [1]. Improvements in image quality have contributed to advances in endoscopic sinus surgery (ESS) and have promoted the use of the endoscopic approach in skull base surgery [2]. Although ESS has shown remarkable progress, there are still problems to overcome; for example, the incidence of perioperative complications has not decreased [3] despite the recent development of new surgical instruments, including endoscopes. Recently, threedimensional (3D) endoscopes became available for use in endonasal surgery, and previous publications have demonstrated the efficacy and limitations of 3D endoscopes in endonasal sinus or skull base surgery [4-7]. In these reports, 3D images were reconstructed from multiple 2D images captured using special lenses that imitate the compound eye of bees, from various angles [8].

In the present study, we examined the efficacy of a 3D endoscope that has two lenses and provides a 3D view from two overlapping 2D images for ESS and skull base surgery.

\section{MATERIALS AND METHODS}

\section{D endoscope}

The 3D endoscope (Shinko Optical, Tokyo, Japan) had an outer diameter of $4.7 \mathrm{~mm}$, 
length $180 \mathrm{~mm}$, and comprised two endoscopes with a 1.9-mm outer diameter for the left and right visual fields (Fig. 1). A circular polarization-multiplexed system was used to generate 3D images. Images from the two lenses were filmed using a high-definition charge-coupled device (CCD) camera at $1920 \times 1080$ pixels, followed by conversion to $1920 \times 540$ pixels. Furthermore, two images were horizontally sliced at the pixel level and visualized on a polarized monitor using the line-by-line method, allowing surgeons to view 3D images through polarized glasses (Fig. 1). A 2D endoscope (Karl Storz, Tuttlingen, Germany) was provided for comparison with the 3D endoscope for specification, visibility, and operability. The specifications of 3D and 2D endoscopes are shown in Table 1. The 2D endoscopes (Medtronic, Minneapolis, USA) were equipped with an irrigation system. In addition, a specially ordered instrument (Yamamura Ikakikai, Kyoto, Japan) which employed the same irrigation system was developed for the 3D endoscope.

\section{Clinical efficacy}

The clinical efficacy of 3D endoscopes was examined during surgical procedures on five cadavers including septoplasty, pansinusotomy, extended sphenoidotomy, frontal sinusotomy with the modified Lothrop procedure, orbital decompression, 
hypophysectomy, and the exposure of the carotid artery. We evaluated the accessibility of the surgical field and image quality. Thereafter, we performed conventional ESS in five cases and endoscopic hypophysectomy in two cases using a 3D endoscope in clinical settings from March 2012 to August 2012. In all cases, a 0²D endoscope was prepared and occasionally used for the comparison of surgical field views between the 3D and 2D endoscopes._All procedures were performed by the same surgeon (T.N.), who verified the visual quality of the surgical fields, operability, and fatigue. In addition, the operative time, amount of bleeding, and frequency of perioperative complications were retrospectively compared with a cohort of control patients who underwent ESS using 2D endoscopes in the Kyoto University Hospital from 2010 to 2012. Statistical comparison was performed using Fisher's exact test regarding the frequency of perioperative complications. In terms of the operative time and amount of bleeding, we investigated whether the results of 3D ESS would fall within the 95\% confidence interval of the data points of 2D ESS. We then compared the results of all the procedures (unilateral multiple sinusotomy, unilateral pansinusotomy, bilateral multiple sinusotomy, and bilateral pansinusotomy). This study was approved by the Ethics Committee of Kyoto University, Japan. 


\section{Educational efficacy}

The educational advantages of the 3D endoscope were assessed using questionnaires given to the participants of cadaver dissection courses at our institution (Kyoto University, Kyoto, Japan). We demonstrated both 3D and 2D endoscopic images to the participants during cadaver dissection for ESS and skull base surgery. The demonstrated procedures included pansinusotomy, extended sphenoidotomy, frontal sinusotomy with the modified Lothrop procedure, orbital decompression, hypophysectomy, and the exposure of the carotid artery. The participants were asked to comment on the following items: (1) Use of 3D endoscopy contributes to a better understanding of surgical anatomy; (2) Use of 3D endoscopy will reduce the risk of perioperative complications; (3) 3D endoscopy facilitates surgical procedures; (4) "Do you want to use a 3D endoscope in clinical settings?" and (5) “At which site would 3D endoscopy be the most useful for understanding surgical anatomy?”

\section{RESULTS}

\section{Clinical efficacy}

During cadaver dissection, all surgical procedures were completed with 3D endoscopy. The viewing angle of the $3 \mathrm{D}$ endoscope used in this study was $80^{\circ}$, which was narrower 
than that of a $2 \mathrm{D}$ endoscope $\left(100^{\circ}\right)$. Narrower viewing angles provided images without distortion and that characteristic led to the precise handling of the endoscope when we operated on lateral structures, such as the uncinate process or the medial wall of the orbit. In the posterior portion of the nasal cavity, images of 3D endoscopy provided a superior perception of depth of information than those via 2D endoscopy. The benefit of 3D viewing was especially obvious in the posterior ethmoid and sphenoid sinuses. Both 3D and 2D endoscopic images of cadavers are shown in Fig. 2.

All endonasal surgeries were completed in clinical settings using a 3D endoscope without perioperative complications. Similar to the results from the cadaver dissections, 3D endoscopy provided a superior perception of depth, especially in the posterior ethmoid and sphenoid sinuses (Fig. 3). In particular, the advantages of 3D viewing were notable during the dissection of a pituitary tumor. The operative time and amount of bleeding associated with 3D ESS and 3D endoscopic hypophysectomy are presented in Table 2A, and the results of 2D ESS are shown in Table 2B. In one of the cases, the operative time was longer than the upper limit of the 95\% confidence interval range in 2D ESS. In the other cases, the operative time was not longer than the upper limit of $95 \%$ confidence interval range of 2D ESS. In terms of the amount of bleeding, all results of 3D ESS fell within the 95\% confidence interval of the results of 2D ESS. However, a disadvantage of 
3D endoscopy was its lack of brightness, which became evident in a bloody surgical field.

There were no particular problems in terms of operability. As for fatigue from 3D viewing through polarized glasses, the surgeon felt eye fatigue at the beginning of the operation; but, after a few minutes, the surgeon's eyes adapted to the field of vision, and eye fatigue did not adversely affect performance of the surgery.

\section{Educational efficacy}

Questionnaires were completed by 73 surgeons, including 63 otolaryngologists and 10 neurosurgeons. The median surgical experience was 14 years (ranging from 2 to 29 years) for the otolaryngologists and 15 years (ranging from 9 to 25 years) for the neurosurgeons. In the questionnaires, $89 \%$ of the respondents agreed that 3D endoscopy provided a better understanding of the surgical anatomy than did 2D endoscopy (Fig. 4). Regarding perioperative complications, $75 \%$ of the respondents stated that 3D endoscopy would decrease the risk of perioperative complications (Fig. 4). In addition, a majority of the respondents wanted to use 3D endoscopy in clinical settings; however, only $50 \%$ of the respondents agreed that 3D endoscopy would make surgical procedures easier (Fig. 4). As for the site where 3D endoscopy would be most useful for understanding surgical anatomy, $40 \%$ of respondents named the skull base; $29 \%$, the posterior ethmoid sinuses; 
26\%, the sphenoid sinus; and 9\%, the ethmoid bulla and middle turbinate.

\section{DISCUSSION}

During surgeries involving conventional 2D endoscopes, it is necessary to understand the complicated 3D structure of the sinuses from planar images. This complexity leads to a lack of perception of depth and causes a disparity between the location recognized on the monitor and the actual location of an instrument, thereby reducing the precision of surgery in the anteroposterior direction. 3D endoscopes were developed in the 1990s as devices meant to help overcome this shortcoming [9]. However, poor image quality and severe visual fatigue have impeded dissemination of 3D endoscopes in clinical practices. Recently, these devices gained popularity among clinicians because of new technological developments. In particular, a laparoscopic operation involving 3D endoscopy has become more common due to the introduction of the da Vinci System (Intuitive Surgical, Sunnyvale, CA, USA) [10]. The dissemination of neuroendoscopy in the field of neurosurgery has accelerated improvements of the endoscopic devices. Thus, at present, 3D endoscopes are similar to 2D endoscopes in size and weight. Regarding their advantages as surgical instruments, several authors have reported the results of endoscopic surgical procedures on the skull base [6-7, 11]. Kari et al. compared the 
operative time, amount of bleeding, duration of hospital stay, and complications between pituitary surgery, involving $2 \mathrm{D}$ and $3 \mathrm{D}$ endoscopy and reported that there were no significant differences [12]. In our results, the operative time of one case was remarkably longer than the results of 2D ESS. However, we considered that mediocre result was caused by assessing the utility of a 3D endoscope during the operative time. Equivalence in bleeding indicated that ESS with a 3D endoscope is not inferior to that with a $2 \mathrm{D}$ endoscope in terms of safety. These results suggest that 3D endoscopic surgery may be an alternative to the conventional 2D endoscopic surgery. Our results also demonstrate a superior perception of depth with 3D endoscopy compared to that with 2D endoscopy; this improvement may ensure more a precise control of surgical instruments. In contrast, the lack of brightness in the surgical view is a disadvantage of 3D endoscopy. This disadvantage does not influence the results of 3D ESS in our experience; however, we suppose that less brightness of 3D endoscope results in the difficulty of operability in the cases with excessive blood loss. Furthermore, blood adhesion on a part of the tip of the 3D endoscope frequently led to the disruption of proper image construction because 3D image synthesis required a clear image from both lenses. Nevertheless, we have developed a special irrigation instrument for the 3D endoscope which applies existing irrigation system. Proper irrigation during surgery enables simultaneous cleaning of the 
lenses of endoscope and cleaning of the bloody surgical field. Therefore, we consider that the advantage of $3 \mathrm{D}$ endoscope becomes if a proper irrigation system is prepared and bleeding is well controlled. In addition, recent developments in monitor and light source technology are remarkable and may contribute to resolving that problem in the near future. With respect to the degree of visual fatigue, Yamauchi et al. assessed the incidence of fatigue while using a stereoscopic endoscope [13]. The results showed no differences in fatigue between 2D and 3D endoscopy during surgeries lasting approximately $1 \mathrm{~h}$. In addition, to prevent eye strain, it is necessary to appropriately set the angle of the lenses and the viewing distance and to reduce the reflection of environmental light on the monitor. We performed surgeries for several hours without fatigue; therefore, the advances in the design of mechanical equipment may have prevented the fatigue that used to be caused by 3D endoscopes. The responses to the questionnaire regarding cadaver dissection with 3D endoscopes show that this method helps to grasp the complicated 3D structure of the sinuses and skull base, especially in posterior locations. These benefits of 3D endoscopy may also help in educating doctors, medical students, and nurses in the operating room.

\section{CONCLUSION}


The 3D endoscope is superior to the $2 \mathrm{D}$ endoscope because the former facilitates an anatomical understanding of posterior structures of the sinuses and skull base. In addition, 3D endoscopy ensures a good precision of surgical manipulations.

Consequently, the 3D endoscope will likely become a standard device for endonasal surgery in the near future.

\section{ACKNOWLEDGMENTS}

This work was supported by a research grant from The Japanese Foundation for

Research and Promotion of Endoscopy.

\section{DISCLOSURE STATEMENT}

The authors report no conflict of interest. 


\section{REFERENCES}

[1] Govindaraj S, Adappa ND, Kennedy DW. Endoscopic sinus surgery: evolution and technical innovations. J Laryngol Otol 2010;124:242-50.

[2] Snyderman CH, Carrau RL, Kassam AB, Zanation A, Prevedello D, Gardner P, et al. Endoscopic skull base surgery: principles of endonasal oncological surgery. J Surg Oncol 2008;97:658-64.

[3] Siedek V, Pilzweger E, Betz C, Berghaus A, Leunig A. Complications in endonasal sinus surgery: a 5-year retrospective study of 2,596 patients. Eur Arch Otorhinolaryngol 2013;270:141-8.

[4] Brown SM, Tabaee A, Singh A, Schwartz TH, Anand VK. Three-dimensional endoscopic sinus surgery: feasibility and technical aspects. Otolaryngol Head Neck Surg 2008;138:400-2.

[5] Tabaee A, Anand VK, Fraser JF, Brown SM, Singh A, Schwartz TH. Threedimensional endoscopic pituitary surgery. Neurosurgery 2009;64:288-93.

[6] Shah RN, Leight WD, Patel MR, Surowitz JB, Wong YT, Wheless SA, et al. A controlled laboratory and clinical evaluation of a three-dimensional endoscope for endonasal sinus and skull base surgery. Am J Rhinol Allergy 2011;25:141-4.. 
[7] Wasserzug O, Margalit N, Weizman N, Fliss DM, Gil Z. Utility of a threedimensional endoscopic system in skull base surgery. Skull Base 2010;20:223-8.

[8] Szold A. Seeing is believing: visualization systems in endoscopic surgery (video, HDTV, stereoscopy, and beyond). Surg Endosc 2005;19:730-3.

[9] Catalano MF, Van Dam J, Bedford R, Cothren RM, Sivak MV Jr. Preliminary evaluation of the prototype stereoscopic endoscope: precise three-dimensional measurement system. Gastrointest Endosc 1993;39:23-8.

[10]Byrn JC, Schluender S, Divino CM, Conrad J, Gurland B, Shlasko E, et al. Threedimensional imaging improves surgical performance for both novice and experienced operators using the da Vinci Robot System. Am J Surg 2007;193:51922.

[11]Manes RP, Barnett S, Batra PS. Utility of novel 3-dimensional stereoscopic vision system for endoscopic sinonasal and skull-base surgery. Int Forum Allergy Rhinol 2011;1:191-7.

[12]Kari E, Oyesiku NM, Dadashev V, Wise SK. Comparison of traditional 2dimensional endoscopic pituitary surgery with new 3-dimensional endoscopic technology: intraoperative and early postoperative factors. Int Forum Allergy Rhinol 2012;2:2-8. 
[13] Yamauchi Y, Shinohara K. Effect of binocular stereopsis on surgical manipulation performance and fatigue when using a stereoscopic endoscope. Stud Health Technol Inform 2005;111:611-4. 
Table 1. Specification of 3D and 2D endoscopes.

\begin{tabular}{|c|c|c|c|c|c|c|}
\hline \multirow[b]{2}{*}{ Endoscope } & \multirow[b]{2}{*}{ Institute } & \multirow[b]{2}{*}{ Length } & \multirow{2}{*}{$\begin{array}{c}\text { Outer } \\
\text { diameter } \\
(\mathrm{mm})\end{array}$} & \multicolumn{2}{|c|}{ Weight (g) } & \multirow{2}{*}{$\begin{array}{c}\text { Viewing } \\
\text { angle }\end{array}$} \\
\hline & & & & Endoscope & Total weight* & \\
\hline $3 D$ & Shinko Optical & 180 & 4.7 & 60 & 465 & $80^{\circ}$ \\
\hline $2 D$ & Karl Storz & 180 & 4.0 & 67 & 370 & $100^{\circ}$ \\
\hline
\end{tabular}

*Weight of endoscope, CCD camera head, and light cable are included in Total weight. 
Table 2. Results of 3D endoscopic sinus surgeries (ESS), 3D endoscopic pituitary surgeries, and 2D ESS.

A. Results of 3D ESS and endoscopic pituitary surgery cases.

\begin{tabular}{|c|c|c|c|c|c|}
\hline Case No. & Age & Sex & Procedure & $\begin{array}{l}\text { Operative } \\
\text { Time (min) }\end{array}$ & $\begin{array}{c}\text { Amount Of } \\
\text { Bleeding }(\mathrm{ml})\end{array}$ \\
\hline 1 & 43 & $\mathrm{~F}$ & unilateral multiple sinusotomy & 103 & 5 \\
\hline 2 & 25 & $\mathrm{M}$ & unilateral multiple sinusotomy & 75 & 5 \\
\hline 3 & 66 & $\mathrm{M}$ & $\begin{array}{c}\text { unilateral pansinusotomy } \\
\text { unilateral multiple sinusotomy }\end{array}$ & 165 & 5 \\
\hline 4 & 64 & $\mathrm{M}$ & bilateral multiple sinusotomy & 71 & 5 \\
\hline 5 & 57 & $\mathrm{M}$ & bilateral pansinusotomy & 128 & 5 \\
\hline 6 & 64 & $\mathrm{~F}$ & hypophysectomy & 339 & 30 \\
\hline 7 & 52 & $\mathrm{~F}$ & hypophysectomy & 222 & 200 \\
\hline
\end{tabular}

B. Results of 2D ESS cases. Average and 95\% confidence interval range of the

operative time and amount of bleeding were calculated for each surgical procedure.

\begin{tabular}{c|cc|cc}
\multirow{2}{*}{ Procedure } & \multicolumn{2}{|c|}{ Operative Time (min) } & Amount Of Bleeding (ml) \\
\cline { 2 - 5 } & Average & $\begin{array}{c}\text { 95\% Confidence } \\
\text { Interval Range }\end{array}$ & Average & $\begin{array}{c}\text { 95\% Confidence } \\
\text { Interval Range }\end{array}$ \\
\hline unilateral multiple sinusotomy ( $\mathrm{n}=38)$ & 68 & $60-75$ & 12 & $7-18$ \\
unilateral pansinusotomy $(\mathrm{n}=8)$ & 72 & $49-96$ & 36 & $0-82$ \\
bilateral multiple sinusotomy ( $\mathrm{n}=14)$ & 95 & $79-110$ & 14 & $0-31$ \\
bilateral pansinusotomy $(\mathrm{n}=18)$ & 147 & $132-162$ & 39 & $7-71$
\end{tabular}




\section{FIGURE LEGENDS}

Fig. 1. A three-dimensional (3D) endoscopy system. The outer diameter (OD) of the 3D endoscope is $4.7 \mathrm{~mm}$, and the endoscope includes two narrow endoscopes (OD: 1.9 mm). Images from the right and left endoscopes are recorded as two $1920 \times 1080$ pixel images. Each image is converted to $1920 \times 540$ pixels and output to a polarized monitor using a line-by-line method. The output image is visualized as a 3D shape by wearing polarized glasses.

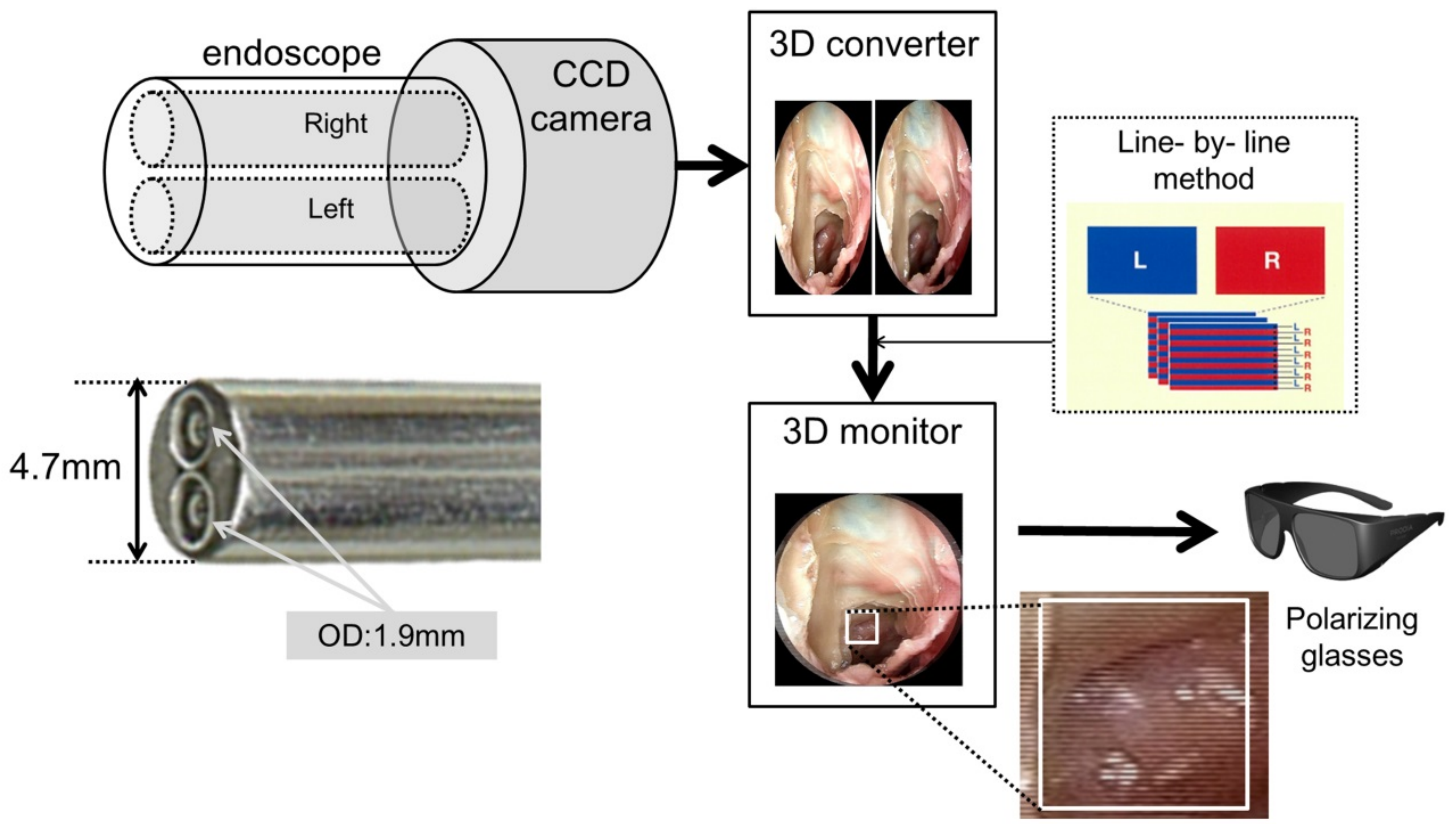


Fig. 2. Comparisons between two-dimensional (2D) and three-dimensional (3D)

endoscopic images. 3D Red-cyan glasses are recommended to view the 3D images

correctly.

A. A 2Dimage of the ethmoidal bulla and middle turbinate (left), and a 3D image of the ethmoidal bulla and middle turbinate (left).

B. 2D and 3D images of the skull base (right). A white arrow indicates anterior ethmoid artery.

C. 2D and 3D images of the posterior ethmoid sinus (right).

D. 2D and 3D images of the sphenoid sinus (right).

E. 2D and 3D images of the pituitary. A white arrow head indicates optico carotid recess.

F. 2D and 3D images of the cavernous sinus.

*, internal carotid artery; UP, uncinate process; EB, ethmoidal bulla; MT, middle turbinate; O, medial wall of the orbit; ST, superior turbinate; OC, optic canal; P, pituitary. 


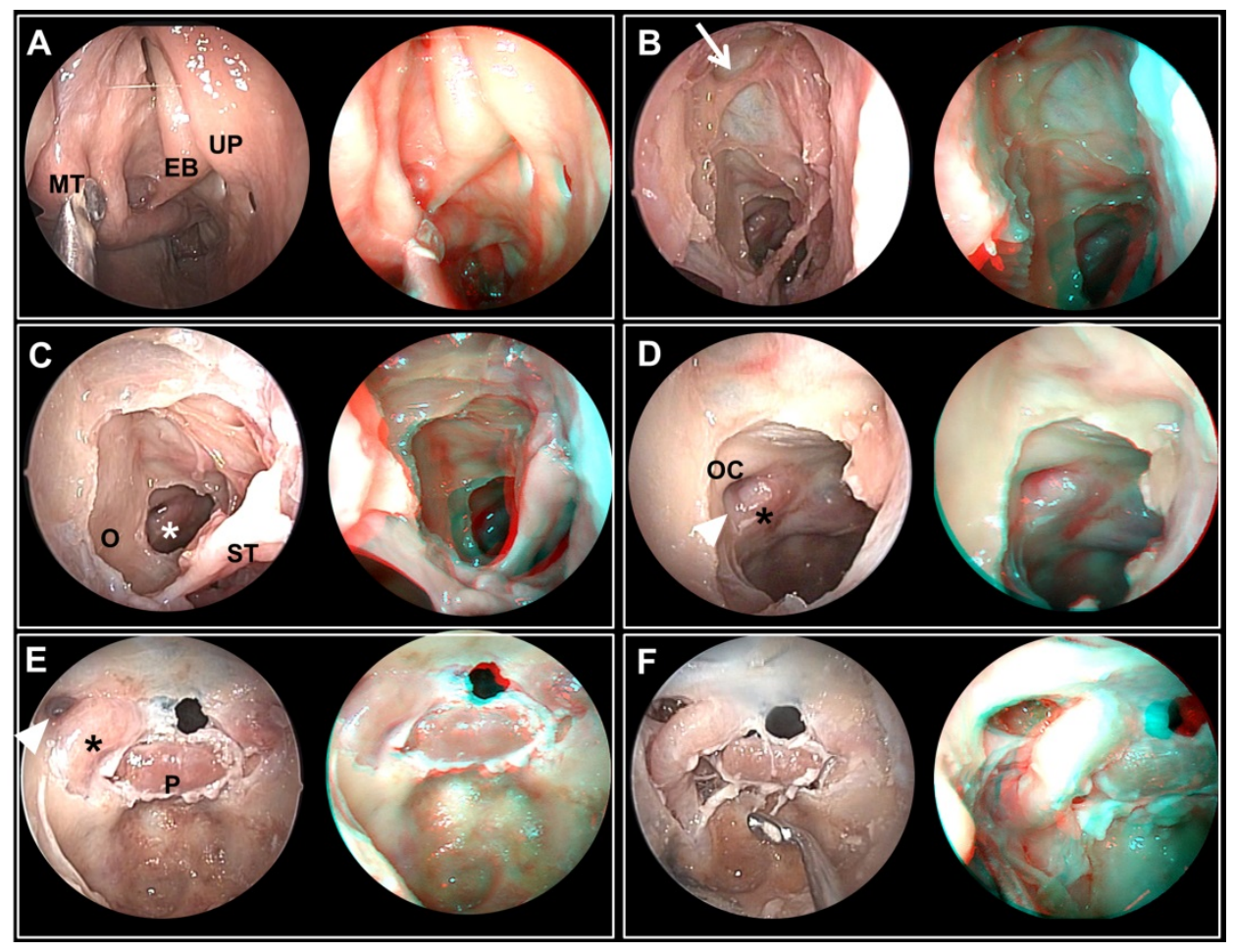

Fig. 3. Three-dimensional (3D) images of the left middle meatus and a pituitary tumor

during surgeries. Red-cyan glasses are recommended to view these images correctly. 3D

images showed superior depth perception toward the posterior ethmoid and sphenoid

sinuses. MS, maxillary sinus; MT, middle turbinate; O, medial wall of the orbit; ST,

superior turbinate; PE, posterior ethmoid sinus; OC, optic canal; T, tumor of the

pituitary gland; SS, sphenoid sinus. 


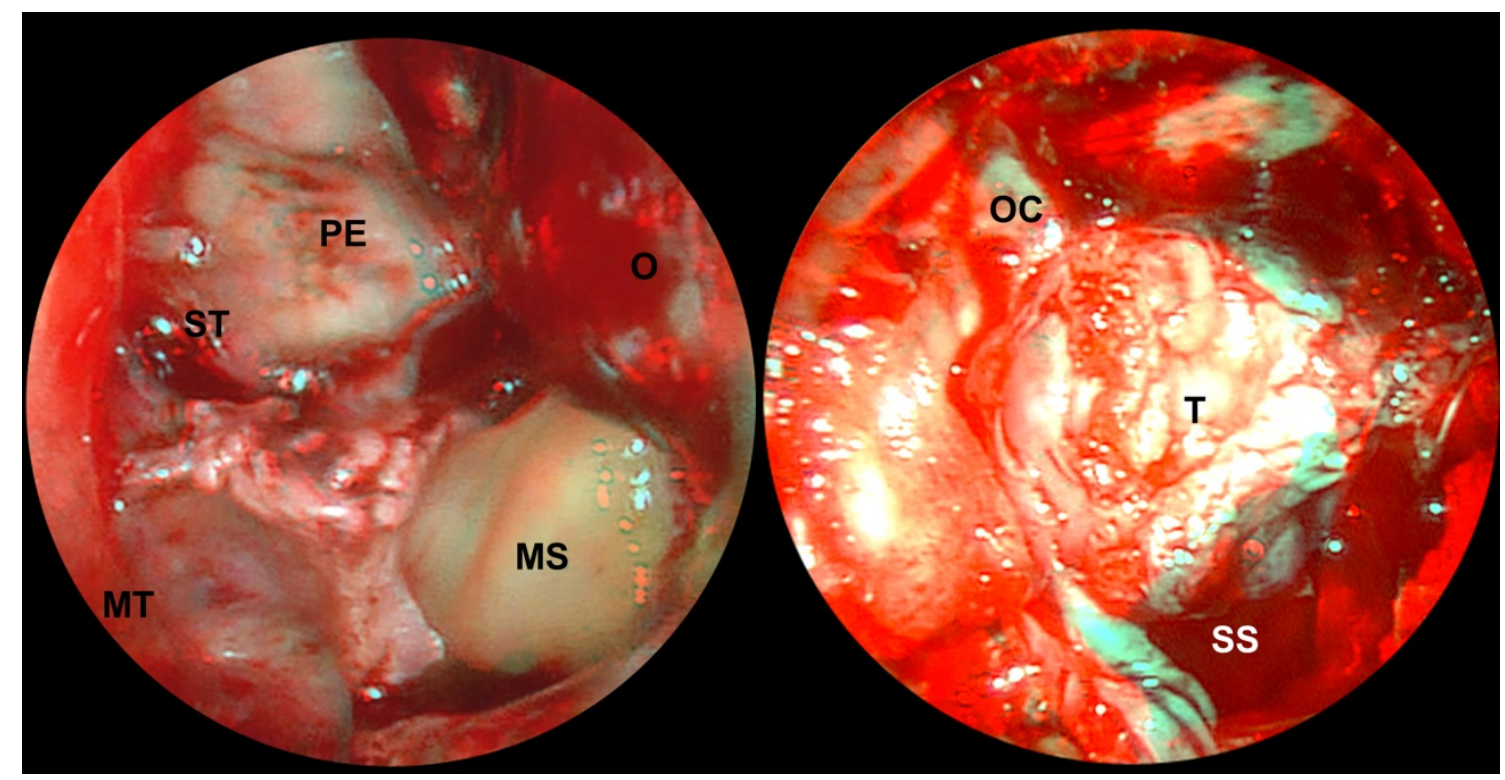

Fig.4. Answers to questionnaires regarding impressions to 3D endoscopic surgery. 73 surgeons, including 63 otolaryngologists and 10 neurosurgeons answered the questionnaires.

- Better understanding the anatomy

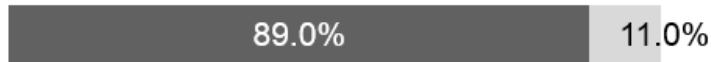

- Decrease complications

\begin{tabular}{ll}
$75.3 \%$ & $24.7 \%$ \\
\hline
\end{tabular}

- Make easy surgical procedures

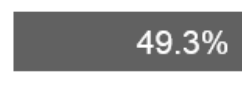

- I want to use 3D in clinical setting

\begin{tabular}{l|l}
$83.6 \%$ & $16.4 \%$
\end{tabular}

